\title{
Brain MRI Characteristics of Patients with Anti-N-Methyl-D- Aspartate Receptor Encephalitis and Their Associations with 2-Year Clinical Outcome
}

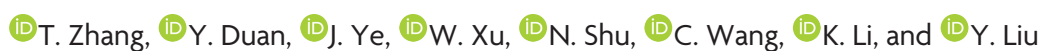

\begin{abstract}
BACKGROUND AND PURPOSE: Anti-N-methyl-D-aspertate receptor encephalitis is an autoimmune-mediated disease without specific brain MRI features. Our aim was to investigate the brain MR imaging characteristics of anti- $N$-methyl-D-aspartate receptor encephalitis and their associations with clinical outcome at a 2-year follow-up.
\end{abstract}

MATERIALS AND METHODS: We enrolled 53 patients with anti- $N$-methyl-D-aspartate receptor encephalitis and performed 2-year follow-up. Brain MRIs were acquired for all patients at the onset phase. The brain MR imaging manifestations were classified into 4 types: type 1: normal MR imaging findings; type 2: only hippocampal lesions; type 3: lesions not involving the hippocampus; and type 4: lesions in both the hippocampus and other brain areas. The modified Rankin Scale score at 2-year follow-up was assessed, and the association between the $\mathrm{mRS}$ and onset brain MR imaging characteristics was evaluated.

RESULTS: Twenty-eight (28/53, 53\%) patients had normal MR imaging findings (type 1), and the others (25/53, 47\%) had abnormal MRI findings: type 2: 7 patients (13\%); type 3: seven patients (13\%); and type 4: eleven patients (21\%). Normal brain MRI findings were more common in female patients $(P=.02)$. Psychiatric and behavioral abnormalities were more common in adults $(P=.015)$, and autonomic symptoms $(P=.025)$ were more common in pediatric patients. The presence of hippocampal lesions $(P=.008, \mathrm{OR}=9.584 ; 95 \% \mathrm{Cl}$, 1.803-50.931) and relapse $(P=.043, \mathrm{OR}=0.111 ; 95 \% \mathrm{Cl}, 0.013-0.930)$ was associated with poor outcome.

CONCLUSIONS: Normal brain MRI findings were observed in half of the patients. Lesions in the hippocampus were the most common MR imaging abnormal finding. The presence of hippocampal lesions is the main MR imaging predictor for poor prognosis in patients with anti-N-methyl-D-aspartate receptor encephalitis.

ABBREVIATIONS: anti-NMDA = anti-N-methyl-D-aspartate; ICU = intensive care unit; MTA = medial temporal lobe atrophy

A nti- $N$-methyl-D-aspartate (anti-NMDA) receptor encephalitis was first described by Dalmau et al in $2007^{1}$ as a common type of autoimmune-mediated limbic encephalitis that presents

Received October 26, 2017; accepted after revision January 14, 2018.

From the Departments of Radiology (T.Z., K.L.) and Neurology (J.Y., W.X.), Xuanwu Hospital, Capital Medical University, Beijing, P.R. China; Department of Radiology (Y.D., Y.L.), Beijing Tiantan Hospital, Capital Medical University, Beijing, P.R. China; Tiantan Image Research Center (Y.D., Y.L.), China National Clinical Research Center for Neurological Diseases, Beijing, P.R. China; State Key Laboratory of Cognitive Neuroscience and Learning and International Data Group/McGovern Institute for Brain Research (N.S., C.W.), Beijing Normal University, Beijing, P.R. China; and Beijing Key Lab of MRI and Brain Informatics (K.L.), Beijing, P.R. China.

Tian Zhang and Yunyun Duan contributed equally to this work.

This work was supported by the European Committee for Treatment and Research in Multiple Sclerosis-Magnetic Imaging in Multiple Sclerosis (ECTRIMS-MAGNMIS) Fellowship from ECTRIMS (Y.L.); the National Science Foundation of China (Nos. 81101038, 81401377, 81471221. and 81230028); the National Basic Research Program of China (2013CB966900); the Beijing Natural Science Fund (No. 7133244); the Beijing Nova Programme (xx2013045); the Beijing Municipal Administration of Hospital Clinical Medicine Development of Special Funding Support (code: ZYLX201609); and Key Projects in the National Science and Technology Pillar Program during the Twelfth Five-Year Plan Period (2012BAl10B04). with various clinical symptoms, including behavioral and psychiatric features, memory and cognitive deficits, seizures, central hypoventilation, and movement disorders. ${ }^{1-5}$ The autoantibody of the anti-NMDA receptor can be detected in the CSF and/or the serum of patients and is a specific antibody that correlates with disease severity. ${ }^{2}$ The management of anti-NMDA receptor encephalitis generally includes immunotherapy and tumor detection and removal. ${ }^{6}$ First-line immunotherapy (eg, steroids, plasmapheresis, and intravenous immunoglobulins) and second-line immunotherapy, including cyclophosphamide or rituximab, are treatment choices that depend on the individual patient status. $^{4,7}$

Please address correspondence to Kuncheng Li, MD, Department of Radiology, Xuanwu Hospital, Capital Medical University, Beijing 100053, China; e-mail: likuncheng@xwh.ccmu.edu.cn; or Yaou Liu, MD, PhD, Department of Radiology, Beijing Tiantan Hospital, Capital Medical University, Beijing, China; Tiantan Image Research Center, China National Clinical Research Center for Neurological Diseases, Beijing, China; e-mail: asiaeurope80@gmail.com

- Indicates open access to non-subscribers at www.ajnr.org

http://dx.doi.org/10.3174/ajnr.A5593 
As reported, normal brain MR imaging results were observed in most patients, ${ }^{8}$ and $33 \%-55 \%$ of patients showed abnormal brain MR imaging results in several previous publications. ${ }^{7,9}$ Brain lesions often occur in the medial temporal lobe, frontal cortex, and parietal cortex, but lesions in the cerebellum, thalamus, basal ganglia, brain

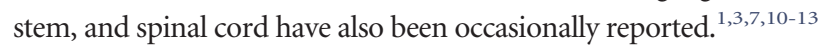
Additionally, pronounced brain atrophy was observed in 2 patients during a 5- to 7-year follow-up. ${ }^{14}$ However, dedicated MR imaging research that focuses on lesion characteristics, especially in Chinese anti-NMDA receptor encephalitis, is lacking, and the associations between the MR imaging characteristics and clinical outcomes remain unknown.

In this study, we systematically investigated the MR imaging features, including lesion location, and the visual rating scale of medial temporal lobe atrophy (MTA $)^{15,16}$ of patients with Chinese anti-NMDA receptor encephalitis and correlated the findings with clinical outcomes as measured by the modified Rankin Scale $^{17}$ at a 2-year follow-up.

\section{MATERIALS AND METHODS \\ Standard Protocol Approvals, Registrations, and Patient Consents}

The institutional review board of Xuanwu Hospital approved the study, and written informed consent was obtained from each participant before participation.

\section{Subjects}

We consecutively enrolled 54 patients in Xuanwu Hospital, Capital Medical University, Beijing, China, from January 2012 to February 2015; one was excluded because of mortality due to cerebrovascular disease. All patients who tested positive for antiNMDA receptor antibodies were diagnosed with anti-NMDA receptor encephalitis and showed typical clinical presentations, the usual course of symptom development,,$^{2,4,18,19}$ as well as CSF and serum that was positive for the associated antibody in tests from 2 independent labs (Tongren Hospital, Capital Medical University, and Peking Union Medical College Hospital in China). Positive antibodies of anti-NMDA receptor in the serum and/or CSF samples were defined according to previously reported criteria, ${ }^{2,9,20}$ including a characteristic pattern of immunostaining of neuropils in the rat brain and specific reactivity with HEK293 cells that express both the NR1 (also named GluN1) and NR2B (also named GluN2B) subunits of the NMDA receptor.

The clinical features were recorded by an experienced neurologist (J.Y, with more than 20 years of experience in neurology). According to the reported manifestations of this disease in previous publications, ${ }^{7,9}$ the symptoms were divided into 4 main groups ${ }^{1}$ : seizures $^{2}$; psychiatric and behavioral features ${ }^{3}$; movement disorders, such as orolingual-facial dyskinesis and uncontrolled motion of the arms and legs ${ }^{4}$; and other symptoms, such as memory deficits, autonomic symptoms (including salivation, urine and/or feces incontinence), central hypoventilation, hemiparesis, and abnormal heart rate. Additionally, the patients were classified into a pediatric group (younger than 18 years of age) or an adult group (18 years of age and older) depending on age.

\section{Brain MR Imaging Acquisition}

Brain MRIs were acquired at a mean time interval of $36 \pm 15$ days (range, 20-90 days) after the presentation onset using a Magnetom Tim Trio or Verio 3T scanner (Siemens, Erlangen, Germany) with an 8-channel phased array head coil in the Department of Radiology, Xuanwu Hospital, Capital Medical University. We obtained the following sequences: axial T2-weighted images (slice $=20$, slice thickness $=5.0 \mathrm{~mm}$, TR/TE $=4040 / 84 \mathrm{~ms}$, flip angle $=160^{\circ}, \mathrm{FOV}=240 \mathrm{~mm}$ ), axial T1-weighted images (slice $=$ 20 , slice thickness $=5.0 \mathrm{~mm}, \mathrm{TR} / \mathrm{TE}=135 / 2.55 \mathrm{~ms}$, flip angle $=$ $50^{\circ}, \mathrm{FOV}=240 \mathrm{~mm}$ ), coronal T1-weighted images (slice $=20$, slice thickness $=5.0 \mathrm{~mm}, \mathrm{TR} / \mathrm{TE}=162 / 3.09 \mathrm{~ms}$, flip angle $=50^{\circ}$, $\mathrm{FOV}=240 \mathrm{~mm}$ ), and fluid-attenuated inversion recovery images (slice $=20$, slice thickness $=5.0 \mathrm{~mm}, \mathrm{TR} / \mathrm{TE}=8500 / 87 \mathrm{~ms}$, flip angle $=150^{\circ}, \mathrm{FOV}=230 \mathrm{~mm}$ ). We defined abnormal brain MR imaging findings as hyperintensity on T2WI and FLAIR and hypointensity on T1WI; we classified abnormalities as hippocampal when hyperintesity or/and volume loss was found in the hippocampus.

Two experienced neuroradiologists (Y.L. and Y.D., both with $>10$ years of experience) assessed brain MR imaging manifestations, which were categorized into 4 types according to the previous reports ${ }^{8}$ after distinguishing constructed defects-type 1: normal MR imaging findings; type 2: only hippocampal lesions; type 3 : lesions not involving the hippocampus; and type 4: lesions in both the hippocampus and other brain areas. Additionally, we measured medial temporal lobe atrophy (Y.L. and Y.D.) by visual assessment on both sides of the brain and used the following 2 categories: normal (MTA = $0-1$ ) and abnormal (MTA $\geq 2$ ). After 3 months, all the images were re-evaluated for MTA scores by the 2 raters. The interrater reproducibility was $98 \%$ and $96 \%$ at the 2 time points of evaluation; the intrarater reproducibility between the 2 raters was $96 \%$ and $94 \%$, respectively.

\section{Clinical Outcome Measurement}

All 53 patients completed the 2-year follow-up. We used the modified Rankin Scale to define the clinical outcome ( good [mRS = $0-1$, recovered well $]$ and poor $[\mathrm{mRS}=2-5$, residual disability $])^{17}$ after immunotherapy treatment according to 2 experienced neurologists (J.Y. and W.X., one with more than 20 years of experience and one with 5 years of experience in neurology).

\section{Statistical Analysis}

Demographic variables including age (pediatric versus adult), sex, and clinical features were analyzed among the groups using the $\chi^{2}$ test. The relationship between baseline MR imaging characteristics and 2-year follow-up mRS scores was evaluated using partial correlation, with age, sex, and time interval (interval between the onset of presentation and MR imaging acquisition) as covariates. We assessed factors that may affect the prognosis by univariate and multivariate binary logistic regression (poor outcome defined as mRS $=2-5$ ), using therapy method (surgery and immunotherapy), intensive care unit (ICU) admission, whether there was a relapse, and hippocampal involvement as factors and using age, sex, and time interval (interval between onset of presentation and MR imaging ac- 
Table 1: Clinical and MRI features of all 53 patients

\begin{tabular}{lccc}
\hline & Pediatric (No.) & Adult (No.) & $\boldsymbol{P}$ Value \\
\hline Sex (female/male) & $10: 7$ & $18: 18$ & .548 \\
$\quad$ Age (range) (median) (yr) & $17(1-17)(15)$ & $36(18-60)(32)$ & - \\
Symptoms & & & \\
$\quad$ Behavioral and psychiatric features & 11 & 33 & .015 \\
Seizure & 10 & 26 & .329 \\
Autonomic symptoms & 3 & 18 & .025 \\
Others & 13 & 29 & .732 \\
Normal/abnormal brain MRI findings & $9 / 8$ & $19 / 17$ & .991 \\
With/without hippocampal involvement & $6 / 11$ & $12 / 24$ & .888 \\
Brain MRI types & & & \\
Type 1 & 9 & 19 & - \\
Type 2 & 2 & 5 & - \\
Type 3 & 2 & 5 & - \\
Type 4 & 4 & 7 & - \\
With/without tumor & $1 / 16$ & $4 / 32$ & .543 \\
Treatment & & & .088 \\
First-line alone & 14 & 32 & - \\
First-line and second-line & 3 & 1 & - \\
Symptomatic treatment & 0 & 3 & - \\
With/without relapse & $5 / 12$ & $5 / 31$ & .178 \\
MTA & & & \\
Normal findings (MTA $=0-1)$ & 14 & 31 & .721 \\
One side/both sides (MTA $\geq 2)$ & 3 & 5 & \\
mRS & & & \\
0-1 & 9 & 13 & .447 \\
$\geq 2$ & 8 & & \\
\hline
\end{tabular}

imab) and 5 patients underwent tumor removal. Three patients received symptomatic treatment only, such as antiepileptic drugs and antipsychotic drugs. Ten patients (19\%) experienced a clinical relapse, which was defined as newonset or worsening symptoms after 2 months of treatment. ${ }^{7}$ During the 2-year follow-up, 21/53 (40\%) patients had residual disability $(\mathrm{mRS}=2-5)$ and 32 $(60 \%)$ patients recovered well $(\mathrm{mRS}=$ 0-1) (Table 1).

\section{Brain MR Imaging Features}

Twenty-eight $(28 / 53,53 \%)$ patients with anti-NMDA receptor encephalitis had normal MR imaging (type 1) results at the onset phase, and 25 patients (25/ $53 ; 47 \%$ ) presented with abnormal MR imaging results - type 2 : seven patients $(7 / 25,28 \%)$ with lesions in the hippocampus only; type 3 : seven patients with lesions in the frontal lobe $(n=3)$; cingulate gyrus and middle cerebellar peduncle $(n=2)$; and corpus callosum, insula, basal ganglia, thalamus, and quisition) as covariates. Statistical analyses were performed using SPSS 23 (IBM, Armonk, New York). The probability map was calculated with SPM8 (http://www.fil.ion.ucl.ac.uk/ $\mathrm{spm} /$ software/spm12) after drawing the ROI.

\section{RESULTS}

\section{Demographic and Clinical Characteristics}

In the final analysis, 53 patients with positive antibody titers and definitively diagnosed with anti-NMDA receptor encephalitis were enrolled and completed the 2-year follow-up. For the antibody, the subtype of antibody and how the type of antibody impacts clinical presentation and brain MR imaging warrant exploration in further studies. Twenty-five male patients (age range, 11-47 years; median age, 26.6 years) and 28 female patients (age range, 12-60 years; median age, 26.2 years) were included. Seventeen patients (32\%) were pediatric patients, and 36 (68\%) were adult patients. Five female patients ( $9 \%, 4$ adults and 1 child) had an underlying neoplasm, and the tumors were pathologically confirmed as ovarian teratomas.

Within the first month of symptom onset, we categorized the symptoms into 4 main groups, and behavioral and psychiatric features $(44 / 53,83 \%)$ represented the most common symptoms. Seizure was the second most common symptom $(36 / 53,68 \%)$. Movement disorders (37/53), memory deficits (10/53), central hypoventilation (9/53), autonomic symptoms (21/53), and other symptoms (13/53) were also observed. The occurrence of behavioral and psychiatric features and autonomic symptoms were different between the 2 age groups (adult: 33/36, 92\%, versus pediatric: $11 / 17,65 \%, P=.015$; and pediatric: $3 / 17,18 \%$, versus adult: $18 / 36,50 \%, P=.025$, respectively) (Table 1 ).

In total, $94 \%$ of patients (50/53) were treated with immunotherapy (steroids and/or intravenous immunoglobulins or ritux- brain stem $(n=1)$, without hippocampal lesions; and type 4: 11 patients $(11 / 25,44 \%)$ with lesions in both the hippocampus and other brain areas, including the frontal lobe and temporal lobes $(n=8)$; parietal lobe $(n=5)$; thalamus $(n=3)$; basal ganglia, cingulate gyrus, and brain stem $(n=2)$; and insula, occipital lobe, corpus callosum, and internal capsule $(n=1)$. One patient had only limbic lesions among 18 patients in types 3 and 4 . The sample images of the $4 \mathrm{MR}$ imaging types are shown in Fig 1, and the lesion probability map is shown in Fig 2. Normal brain MR imaging results were observed more frequently in female patients (19 of $28,68 \%)$ than in male patients ( 9 of $25,36 \%)(P=.020)$. No difference was observed in brain MR imaging manifestations between age groups (pediatric versus adult groups) $(P=.982)$.

Forty-five patients had a normal MTA score $($ MTA $=0-1)$ on both sides of the brain, 2 patients showed atrophy $(\mathrm{MTA} \geq 2)$ on both sides, and 6 patients showed atrophy in only 1 side: on the right side in 2 patients (median MTA score $=2$ ) and on the left side in 4 patients (median MTA score: $=2$ ).

\section{Association between MR Imaging Characteristics and Clinical Outcome}

The patients with hippocampal lesions had higher mRS scores than those without hippocampal lesions after taking age, sex, and time interval as covariates $(P=.032)$. Partial correlation analyses showed that the MTA score of the left side was correlated with the mRS score at 2-year follow-up $(r=0.319, P=.020)$. In univariate and multivariate logistic regression, both relapse $(P=.040$ versus $P=.043)$ and involvement of the hippocampus $(P=.025$ versus $P=.008)$ were associated with clinical outcome. Additionally, hippocampal involvement $(P=.008$; OR $=9.584$; 95\% CI, $1.803-$ $50.931)$ had a stronger association with clinical outcome than 

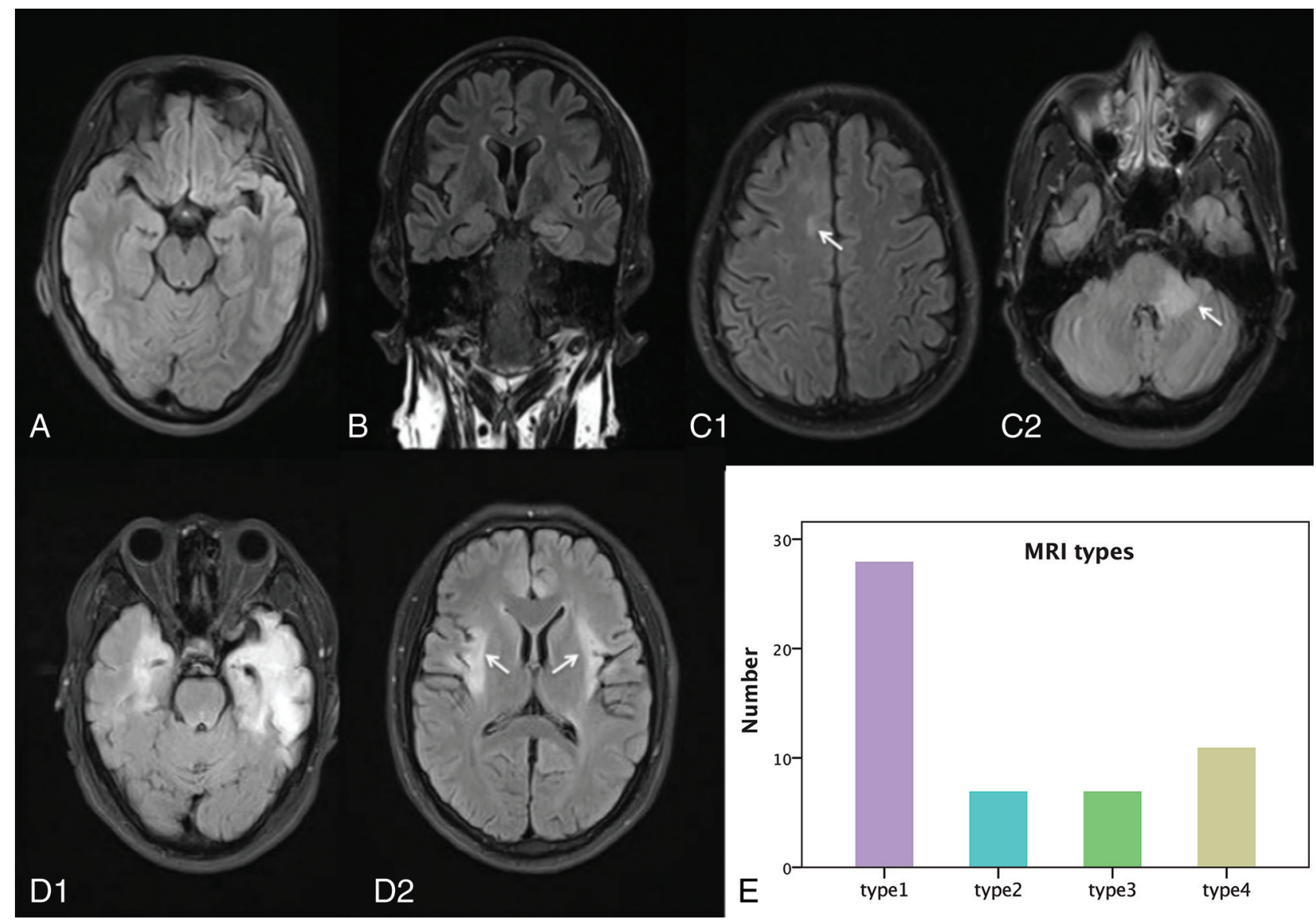

FIG 1. Four types of brain MR imaging appearances in patients with anti-NMDA receptor encephalitis, and the histogram of the 4 types of brain MR imaging appearance. Axial $(A, C$, and $D)$ and coronal FLAIR images $(B)$ come from 4 patients ( $C 7$ and $C 2$ from same patient, $D 1$ and $D 2$ from same patient). A, Type 1, a 23-year-old male patient with anti-NMDA receptor encephalitis, with normal brain MR imaging findings. $B$, Type 2, a 29-year-old female patient. Lesions are in the left hippocampus with bilateral mild volume loss in the hippocampus. C, Type 3, a 28-year-old male patient. Lesions are in the right frontal lobe (white arrow) and middle cerebellar peduncle (white arrow) and brain stem. D, Type 4, a 25-year-old male patient, with lesions located in the bilateral frontal lobe, temporal lobe, insula (white arrows), hippocampus, and cingulate gyrus, with volume loss in the left hippocampus. $E$, Histogram of the 4 types of brain MR imaging appearances.

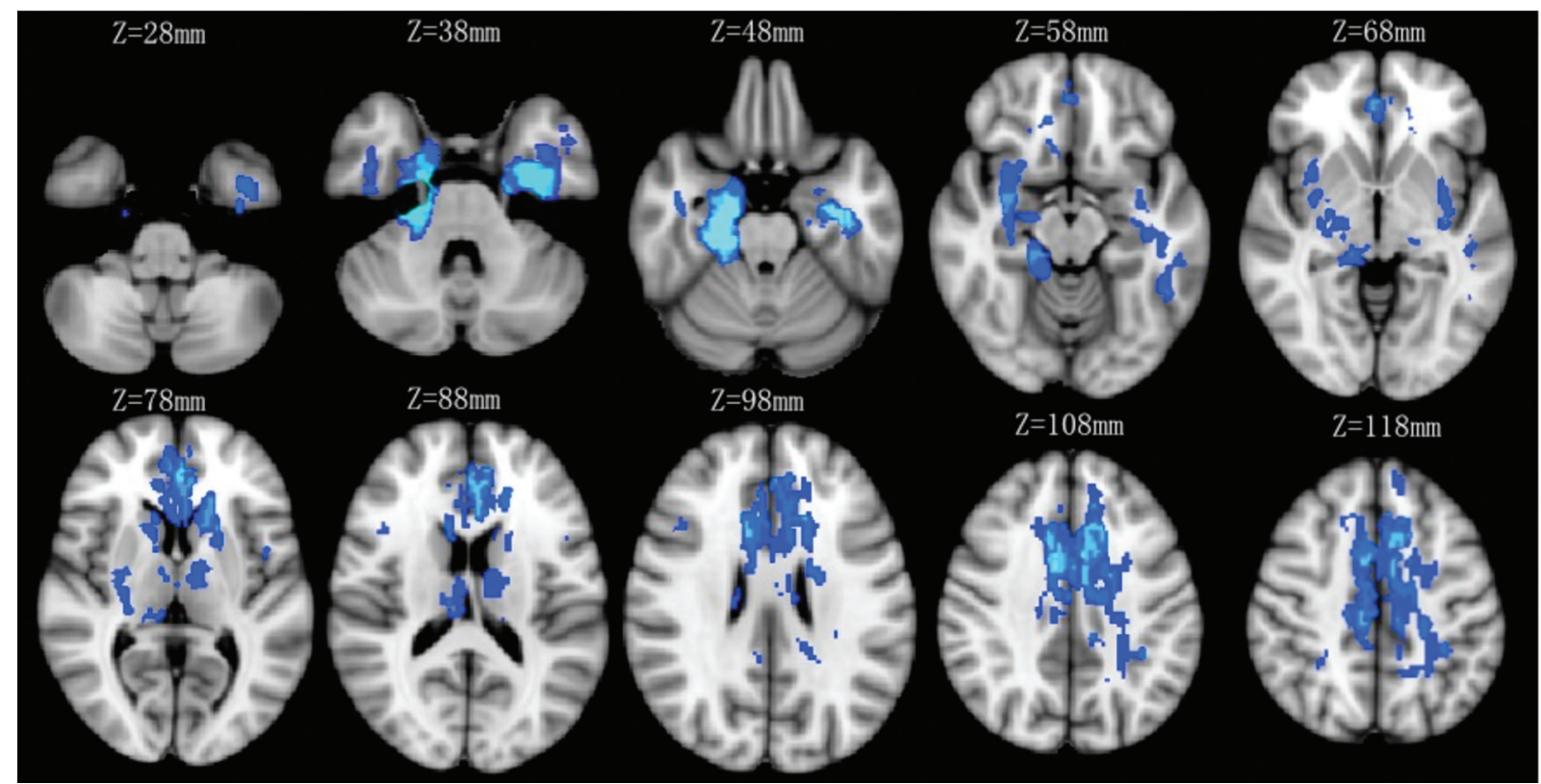

FIG 2. Lesion probability map of brain MR imaging. The mean lesion probability distribution thresholded at $34 \%$ is shown in light blue and thresholded at $1 \%$ is shown in blue and overlaid on the ICBM152 T7 template in the Montreal Neurological Institute space. 
Table 2: Factors associated with poor prognosis (mRS $\geq 2$ )

$P$ Value Odds Ratio $(95 \% \mathrm{Cl})$

\begin{tabular}{|c|c|c|}
\hline \multicolumn{3}{|l|}{ Univariate analysis $^{\mathrm{a}}$} \\
\hline Hippocampal involvement & .025 & 3.929 (1.185-13.021) \\
\hline Relapse & .040 & $0.316(0.060-1.665)$ \\
\hline Treatment & .753 & 1.389 (0.180-10.735) \\
\hline Admitted to ICU & .352 & $0.500(0.116-2.155)$ \\
\hline Teratoma & .999 & - \\
\hline \multicolumn{3}{|l|}{ Multivariate analysis ${ }^{\mathrm{a}, \mathrm{b}}$} \\
\hline Hippocampal involvement ${ }^{c}$ & .008 & $9.584(1.803-50.931)$ \\
\hline Relapse & .043 & $0.111(0.013-0.930)$ \\
\hline Treatment & .753 & $1.389(0.180-10.735)$ \\
\hline Admitted to ICU & .592 & $0.636(0.121-3.334)$ \\
\hline
\end{tabular}

a Using binary logistic regression.

${ }^{b}$ Analyzed using the therapy method (operation and immunotherapy), ICU admission, and whether there was relapse and hippocampal involvement as factors and age, sex, and time interval (interval between onset of presentation and MRI acquisition) as covariates.

'Hippocampal involvement including both types 2 and 4.

relapse $(P=.043$; OR $=0.111 ; 95 \% \mathrm{CI}, 0.013-0.930)$. No correlation was observed between clinical outcome and intensive care unit admission, treatment, or teratoma (Table 2).

\section{DISCUSSION}

In this study, we demonstrated the MR imaging features of patients with Chinese anti-NMDA receptor encephalitis and their associations with clinical outcomes: 1) We categorized the brain MR imaging of anti-NMDA receptor encephalitis into 4 types and normal brain MR imaging acquisition findings accounted for $>50 \%$ of cases. Normal brain MR imaging results were observed more frequently in female patients than in male patients. 2) Hippocampal lesions were the most common brain abnormality, and 3) hippocampal lesions and relapse were risk factors that contributed to a poor prognosis.

For demographic and clinical characteristics, the age distribution (range, 11-60 years; mean age, 26.4 years) in our study is similar to that in previous anti-NMDA receptor encephalitis studies, ${ }^{7,20,21}$ but the proportion of male patients is higher (47\%) compared with previous studies. ${ }^{2,3,7,8}$ This divergence of sex differences between Chinese and Western patients may be due to racial differences or the relatively small sample size in our study. Therefore, future studies with larger samples are required to compare the demographic features between Chinese and Western patients. The clinical pattern and evolution are similar between adult and pediatric patients. ${ }^{22,23}$ For clinical symptoms, behavioral and psychiatric features were observed in the largest proportion of patients and are the main causes for admission to the hospital. Behavioral and psychiatric features and autonomic symptoms appeared in adults more frequently than in pediatric patients, which is consistent with previous research. ${ }^{3}$ Clinical relapse appeared in 10 patients (19\%), which is similar to the $12 \%-$ $24 \%$ frequency of neurologic relapse observed in previous studies. $^{7,8,24}$

Normal and abnormal brain MR imaging results were observed in 28 and 25 patients with the anti-NMDA receptor encephalitis, respectively; this finding is consistent with those in previous publications. ${ }^{1,2,11}$ In female patients, normal brain MR imaging findings were encountered more often in than male patients, implying the different lesion patterns between sexes. Fur- ther studies with advanced MR imaging techniques such as diffusion imaging and functional MR imaging should be conducted in the patients with normal routine brain MR imaging findings to reveal the microstructural and functional changes. ${ }^{25}$ In patients with abnormal brain MR imaging findings, lesions can occur in various locations such as the hippocampus, thalamus, basal ganglia, brain stem, insula, and frontal, temporal, and parietal lobes; and white matter lesions can occur in the corpus callosum, a finding reported by other studies. ${ }^{1,3,10-13}$ In the current study, to simplify the brain MR imaging patterns and characteristics, we classified brain MR imaging appearance into 4 types: type 1 (normal), type 2 (only hippocampal lesion), type 3 (lesions in other brain areas), and type 4 (hippocampal lesion and lesions in other areas). In these 3 abnormal types, type 4 was the most common pattern $(21 \%)$, implying that lesions in other locations often occur together with hippocampal lesions in patients with anti-NMDA receptor encephalitis.

The hippocampus was the most common lesion site in patients with Chinese anti-NMDA receptor encephalitis, which was observed in approximately one-third of the patients. Previous studies have shown that structural hippocampal damage represented the target change in patients with anti-NMDA receptor encephalitis, ${ }^{25,26}$ which was significantly related to memory deficits and disease severity. Additionally, lesions located in the frontal and temporal lobes were present in approximately $15 \%$ of patients $(21 \%$ and $15 \%)$, suggesting that the frontal and temporal lobes were the second most common lesion site after the hippocampus.

Medial temporal lobe atrophy as measured by MTA was observed in 8 patients $(15 \%)$ on at least 1 side, indicating that structural damage in the hippocampus was relatively common. MTA of the left side was correlated with the mRS; this finding is consistent with that in a previous study showing that left hippocampal volume could predict disease severity. ${ }^{26}$ The findings suggest MTA as a valuable biomarker for predicting disease severity.

Poor prognosis was correlated with clinical relapse and hippocampal lesions in the present study, and hippocampal involvement had a stronger association with clinical outcome than relapse. Admission to an ICU, the method of treatment, and teratoma had no relationship with the clinical outcome. In several previous studies, some other factors were reported to be associated with clinical outcomes, including lack of a need for admission to an intensive care unit, low severity of the disease within 4 weeks, ${ }^{7}$ and a low titer of serum and CSF NMDA receptor antibodies, ${ }^{8,9,27}$ which were not identified in our study. The discordance between our study and some previous studies was due to the small number of patients, the different definitions of good prognosis, ${ }^{7}$ or the lack of an early mRS assessment.

\section{Limitations}

Our study has some limitations. First, the sample size was relatively small, and the significance and reliability of the results need to be improved by enrolling more subjects. Additionally, the data were obtained from a single center. Further studies should focus on multicenter samples to verify the results. Second, brain MR imaging was conducted only at the onset phase, so the MR imaging changes of this disease at different phases or changes with 
other auxiliary examinations are not clear. In this study, only routine sequences with slice thicknesses of $5 \mathrm{~mm}$ on brain MR imaging were obtained, on which it is difficult to detect small lesions and accurately measure the brain and hippocampal volumes. For MTA score assessment, only 79\% of the patients had coronal images. A comprehensive scan and advanced MR imaging techniques with high resolution such as functional MR imaging should be conducted in these patients. ${ }^{28}$ Third, the assessment of clinical outcome in this study included only mRS scores at the 2-year follow-up. A more systematic assessment ${ }^{29}$ should be performed in a future study.

\section{CONCLUSIONS}

In patients with Chinese anti-NMDA receptor encephalitis, half had normal brain MR imaging findings, and normal brain MRI findings were more common in female patients. Psychiatric and behavioral abnormalities were more common in adults, and autonomic symptoms were more common in pediatric patients. Hippocampal lesions were the most common MR imaging abnormal finding. The presence of hippocampal lesions and relapse were major factors in the prediction of a poor prognosis.

\section{ACKNOWLEDGMENTS}

The authors thank Dr Kristofer Wood from Barrow Neurological Institute for language revision and Kun Yang from Xuanwu Hospital, Capital Medical University, for statistics review. The authors also thank the patients in this study and members of Neuroimmunology Team and staff of the Department of Radiology for various kinds of support, especially Drs Jing Huang and Zhuoqiong Ren.

\section{REFERENCES}

1. Dalmau J, Erdem T, Wu H-y, et al. Paraneoplastic anti-N-methylD-aspartate receptor encephalitis associated with ovarian teratoma. Ann Neurol 2007;61:25-36 CrossRef

2. Dalmau J, Lancaster E, Martinez-Hernandez E, et al. Clinical experience and laboratory investigations in patients with anti-NMDAR encephalitis. Lancet Neurol 2011;10:63-74 CrossRef Medline

3. Viaccoz A, Desestret V, Ducray F, et al. Clinical specificities of adult male patients with NMDA receptor antibodies encephalitis. Neurology 2014;82:556-63 CrossRef Medline

4. Jones KC, Benseler SM, Moharir M. Anti-NMDA receptor encephalitis. Neuroimaging Clin N Am 2013;23:309-20 CrossRef Medline

5. Finke C, Kopp UA, Prüss $\mathrm{H}$, et al. Cognitive deficits following antiNMDA receptor encephalitis. J Neurol Neurosurg Psychiatry 2012;83: 195-98 CrossRef Medline

6. Gultekin SH, Rosenfeld MR, Voltz R, et al. Paraneoplastic limbic encephalitis: neurological symptoms, immunological findings and tumour association in 50 patients. Brain 2000;123:1481-94 CrossRef Medline

7. Titulaer MJ, McCracken L, Gabilondo I, et al. Treatment and prognostic factors for long-term outcome in patients with anti-NMDA receptor encephalitis: an observational cohort study. Lancet Neurol 2013;12:157-65 CrossRef Medline

8. Irani SR, Bera K, Waters $\mathrm{P}$, et al. N-methyl-D-aspartate antibody encephalitis: temporal progression of clinical and paraclinical observations in a predominantly non-paraneoplastic disorder of both sexes. Brain 2010;133:1655-67 CrossRef Medline

9. Dalmau J, Gleichman AJ, Hughes EG, et al. Anti-NMDA-receptor encephalitis: case series and analysis of the effects of antibodies. Lancet Neurol 2008;7:1091-98 CrossRef Medline
10. Greiner H, Leach JL, Lee KH, et al. Anti-NMDA receptor encephalitis presenting with imaging findings and clinical features mimicking Rasmussen syndrome. Seizure 2011;20:266-70 CrossRef Medline

11. Günther A, Brodoehl S, Witte OW, et al. Atypical posthypoxic MRI changes in hypermetabolic regions in anti-NMDA-receptor encephalitis. Neurology 2012;79:720-21 CrossRef Medline

12. Hoffmann LA, Jarius S, Pellkofer HL, et al. Anti-Ma and anti-Ta associated paraneoplastic neurological syndromes: 22 newly diagnosed patients and review of previous cases. J Neurol Neurosurg Psychiatry 2008;79:767-73 CrossRef

13. Haberlandt E, Bast T, Ebner A, et al. Limbic encephalitis in children and adolescents. Arch Dis Child 2011;96:186-91 CrossRef Medline

14. Iizuka T, Yoshii S, Kan S, et al. Reversible brain atrophy in antiNMDA receptor encephalitis: a long-term observational study. J Neurol 2010;257:1686-91 CrossRef Medline

15. Wahlund LO, Julin P, Johansson SE, et al. Visual rating and volumetry of the medial temporal lobe on magnetic resonance imaging in dementia: a comparative study. J Neurol Neurosurg Psychiatry 2000; 69:630-35 CrossRef Medline

16. Bresciani L, Rossi R, Testa C, et al. Visual assessment of medial temporal atrophy on MR films in Alzheimer's disease: comparison with volumetry. Aging Clin Exp Res 2005;17:8-13 CrossRef Medline

17. van Swieten JC, Koudstaal PJ, Visser MC, et al. Interobserver agreement for the assessment of handicap in stroke patients. Stroke 1988; 19:604-07 CrossRef Medline

18. Peery HE, Day GS, Dunn S, et al. Anti-NMDA receptor encephalitis: the disorder, the diagnosis and the immunobiology. Autoimmun Rev 2012;11:863-72 CrossRef Medline

19. Maneta E, Garcia G. Psychiatric manifestations of anti-NMDA receptor encephalitis: neurobiological underpinnings and differential diagnostic implications. Psychosomatics 2014;55:37-44 CrossRef Medline

20. Sansing LH, Tüzün E, Ko MW, et al. A patient with encephalitis associated with NMDA receptor antibodies. Nat Clin Pract Neurol 2007;3:291-96 CrossRef Medline

21. Parratt KL, Allan M, Lewis SJ, et al. Acute psychiatric illness in a young woman: an unusual form of encephalitis. Med J Aust 2009; 191:284-86 Medline

22. Florance NR, Davis RL, Lam C, et al. Anti-N-methyl-D-aspartate receptor (NMDAR) encephalitis in children and adolescents. Ann Neurol 2009;66:11-18 CrossRef Medline

23. Lin JJ, Lin KL, Hsia SH, et al; Children with Encephalitis and/or Encephalopathy Related Status Epilepticus and Epilepsy (CHEESE) Study Group. Anti-N-methyl-D-aspartate receptor encephalitis in Taiwan: a comparison between children and adults. Pediatr Neurol 2014;50:574-80 CrossRef Medline

24. Gabilondo I, Saiz A, Galán L, et al. Analysis of relapses in antiNMDAR encephalitis. Neurology 2011;77:996-99 CrossRef Medline

25. Finke C, Kopp UA, Scheel M, et al. Functional and structural brain changes in anti-N-methyl-D-aspartate receptor encephalitis. Ann Neurol 2013;74:284-96 CrossRef Medline

26. Finke C, Kopp UA, Pajkert A, et al. Structural hippocampal damage following anti-N-methyl-D-aspartate receptor encephalitis. Biol Psychiatry 2016;79:727-34 CrossRef Medline

27. Gresa-Arribas N, Titulaer MJ, Torrents A, et al. Antibody titres at diagnosis and during follow-up of anti-NMDA receptor encephalitis: a retrospective study. Lancet Neurol 2014;13:167-77 CrossRef Medline

28. Baumgartner A, Rauer S, Mader I, et al. Cerebral FDG-PET and MRI findings in autoimmune limbic encephalitis: correlation with autoantibody types. J Neurol 2013;260:2744-53 CrossRef Medline

29. Peer M, Prüss H, Ben-Dayan I, et al. Functional connectivity of large-scale brain networks in patients with anti-NMDA receptor encephalitis: an observational study. Lancet Psychiatry 2017;4: 768-74 CrossRef Medline 